\title{
Análisis De Representaciones Gráficas Cartesianas En Libros De Texto De Biología
}

\section{Eugenia Cristina Artola, Profesor Adjunto}

Departamento Matemática, Facultad de Ingeniería. Universidad de Mendoza, Argentina

Liliana Esther Mayoral Nouveliere, Profesor Adjunto

Departamento de Formación Docente, Facultad de Ciencias Exactas y

Naturales, Universidad Nacional de Cuyo, Mendoza, Argentina Alicia Benarroch Benarroch, Profesora Titular

Departamento de Didáctica de las Ciencias Experimentales, Facultad de

Educación y Humanidades. Universidad de Granada, Melilla, España

doi: 10.19044/esj.2016.v12n36p109 URL:http://dx.doi.org/10.19044/esj.2016.v12n36p109

Abstract

Graphical representations Cartesian (RGC) are important communication tools in different areas of knowledge and in particular in the Population Biology (BP). The teaching of this area can be strongly affected by the difficulties that students encounter in the interpretation and use of the RGC. In this paper the analysis of RGC in ten textbooks and eight Secondary Education Teacher Training Biology, most widespread in the Province of Mendoza (Argentina), intended to compare and contrast their results is made. The analysis of character ontosemiotic involves both the text and the images, and uses a set of indicators associated with the semiotic concepts of relevance, appropriateness and didactic suitability, based on previous research. We conclude that the books of Teacher Education use a greater amount of RGC and generate as many positive interactions in their school books readers. They are therefore most flattering of inter-linguistic semiotic interactions and relationships between mathematical and biological language. However, they are a source of potential conflict, like school, largely because of lack of clarification of meanings of symbolic notations.

Keywords: Text analysis. Graphical representation cartesian. Population Biology. High School. Teacher training.

\section{Resumen}

Las Representaciones Gráficas Cartesianas (RGC) son instrumentos de comunicación importantes en distintos ámbitos de conocimientos y en 
particular en el de la Biología de Poblaciones (BP). La enseñanza de esta área puede verse fuertemente afectada por las dificultades que los estudiantes encuentran en la interpretación y uso de las RGC. En este trabajo se realiza el análisis de las RGC en diez libros de texto de Educación Secundaria y en ocho de Formación de Profesorado de Biología, más frecuentes en instituciones de la Provincia de Mendoza (Argentina), con la intención de comparar y contrastar sus resultados. El análisis, de carácter ontosemiótico, implica tanto al texto como a las imágenes, y utiliza un conjunto de indicadores asociados a los conceptos semióticos de pertinencia, adecuación e idoneidad didáctica, fundamentados en investigaciones previas. Se concluye que los libros de Formación del Profesorado utilizan una mayor cantidad de RGC, generando mayor frecuencia de interacciones positivas en sus lectores que los libros escolares. Son por tanto más favorecedores de las interacciones semióticas inter-lingüísticas, y de las relaciones entre el lenguaje matemático y biológico. No obstante, son fuente de conflictos potenciales, al igual que los escolares, principalmente por la falta de aclaración de significados de notaciones simbólicas.

Palabras clave. Libros de texto. Representaciones gráficas cartesianas. Biología de poblaciones. Educación Secundaria. Formación del Profesorado.

\section{Introducción}

Vivimos en una cultura visual repleta de representaciones gráficas, usadas como herramientas para comunicar ideas y fenómenos. La dialéctica entre la realidad y la representación se remonta a los comienzos de la palabra escrita, cuando ya la tecnología alfabética desarticuló la captación meramente auditiva de la palabra para convertirla en visual, produciendo con ello un cambio fundamental en el significado. Las representaciones, según Postigo y Pozo (1999), pueden ser de diferentes tipos: diagramas, ilustraciones, gráficas cartesianas, mapas, esquemas, entre otras. En el caso particular de la enseñanza de las Ciencias Biológicas, la iconografía en general, y las representaciones gráficas cartesianas (RGC) en particular, pueden ser muy útiles para expresar y comunicar las relaciones entre dos o más variables (Jiménez Tejada, 2009), analizar su variación (Arias, Leal y Organista, 2011), así como la transformación de significados cuando se opta por distintas escalas o niveles organizacionales.

La Biología de Poblaciones (BP) se sostiene en una serie de conceptos, procedimientos y actitudes que impactan de modo muy directo en la construcción de competencias vinculadas con la interacción del estudiante en el medio ambiente. La dinámica de poblaciones se puede representar mediante modelos matemáticos en los cuáles se relacionan variables, por ejemplo: los cambios de poblaciones en distintas escalas y valores; el espacio 
y el número de individuos; la sucesión en distintos tiempos; la estructura genética de poblaciones o la composición de especies en comunidades biológicas. Por ello, en la enseñanza y aprendizaje de conceptos biológicos se hace necesario desarrollar no sólo el dominio de dichos conceptos, sino también la habilidad de representarlos utilizando diferentes formas (Arias et al., 2011) entre las que se destacan las RGC, ya que constituyen una forma habitual de comunicación científica y una herramienta útil para el trabajo didáctico en este contenido (García, 2005).

Las RGC son aquéllas que expresan una relación numérica entre dos variables a través de distintos elementos espaciales: barras, líneas, puntos, etc. Frecuentemente los novatos tienden a realizar un procesamiento muy ligero y en la mayoría de los casos se limitan a la lectura de los datos y al reconocimiento de aspectos puntuales de las RGC, apareciendo dificultades cuando se indaga en niveles más profundos de interpretación de la información representada (Preece y Janvier, 1993; González Astudillo, 2004; Pozo y Flores, 2007), o cuando se solicita su construcción. Es posible que estas dificultades estén interfiriendo en la falta de diferenciación de diversos conceptos estructurantes de ecología, como población, comunidad y especie (Jiménez Tejada et al, 2013).

El mundo orgánico es tan matemático como el inorgánico y muchas de las dificultades del aprendizaje de las nociones biológicas podrían estar vinculadas a la necesidad de comprender las herramientas matemáticas usadas en su enseñanza. La naturaleza del lenguaje matemático es entendida de formas muy diversas entre docentes y alumnos, dificultando de esta manera su comunicación y su aprehensión. Si bien, hasta cierto punto, nadie deja nunca de aprender un lenguaje, a medida que nuestro sentido del control y dominio de estos sistemas aumenta a la luz de experiencias más amplias y profundas, su riqueza no es frecuentemente utilizada con fines didácticos en las clases ni en las producciones escritas, es por esto que el estudio de la naturaleza del lenguaje matemático y de los principios y reglas que lo rigen tiene un valor fundamental aportando elementos importantes para la práctica escolar en sí, así como para el diseño de los materiales escritos (Pimm, 1990).

Interpretar, desde la lectura a la construcción, la RGC reclama no solamente situaciones matemáticas, sino pertenecientes a un determinado contexto, en este caso en particular al de BP. Lo ideal sería que los conceptos matemáticos ayudaran a comprender los fenómenos estudiados en la clase de Biología y que éstos a su vez enriquecieran a los primeros. Sin embargo, existe una falta de interconexión curricular entre Matemática y Biología y ello puede acarrear inconvenientes en el proceso de la construcción social del conocimiento, pues no es usual presentar al estudiante los conocimientos de las diferentes áreas de manera holística sino desagregada (Jiménez Tejada et al, 2008). 
Este análisis de textos forma parte de un trabajo de investigación en el que se indaga acerca de la enseñanza y aprendizaje de las RGC en el dominio de la BP, área en la que son abundantes los conceptos que relacionan dos o más variables, como tasas de crecimiento poblacional, de migración, de natalidad, de morbimortalidad, de frecuencias feno o genotípicas entre otros, $\mathrm{y}$ en la que frecuentemente se utilizan las RGC para comunicar dichas relaciones. Las propuestas curriculares, de alcance nacional en Argentina y provincial en Mendoza, señalan la necesidad de potenciar en los estudiantes la formación de capacidades y competencias en el desempeño como ciudadanos en interacción con el medio ambiente. Esto nos condujo a indagar acerca de las propuestas para el desarrollo de saberes, de las funciones cognitivas y de las actividades ligadas a la semiosis implicadas en los procesos de enseñanzaaprendizaje de conceptos de BP vinculados a las RGC. La meta fue obtener señales concretas de aporte teórico-didáctico a partir de resolver un análisis de tipo ontosemiótico sobre las propuestas para la interacción de los alumnos con las RGC en el contexto de BP, presentes en los textos para la Educación Secundaria y Formación de Profesorado en Ciencias Biológicas.

\section{Objetivos}

Alcanzar la meta, reclama determinar objetivos específicos que permitan advertir en las propuestas editoriales, del área de las Ciencias Naturales en general o del campo de la Biología especialmente, para los diferentes niveles educativos (educación secundaria y de formación docente) los indicadores del enfoque ontosemiótico y la respectiva contrastación. Por ello se determinó:

- $\quad$ Analizar, desde un enfoque ontosemiótico, las $R G C$ vinculadas a BP de los libros de textos de Ciencias Naturales de $1^{\circ}$ y $2^{\circ}$ año de Educación Secundaria más frecuentes en las bibliotecas escolares de la ciudad de Mendoza, Argentina.

- $\quad$ Aplicar el análisis ontosemiótico anterior, a las $R G C$ vinculadas a BP de los libros de texto de Biología y Ecología, que más se utilizan en la Formación del Profesorado en Biología en la provincia de Mendoza.

- $\quad$ Comparar los resultados del análisis ontosemiótico realizado entre las propuestas editoriales de ambos niveles educativos.

\section{Bases Teóricas \\ El libro de texto ${ }^{1}$, un recurso didáctico}

\footnotetext{
1 Término que designa aquellos manuales que utilizan habitualmente profesores y alumnos a lo largo del curso escolar en el proceso de enseñanza-aprendizaje de un área de conocimiento (González Astudillo y Sierra, 2004).
} 
Los libros de textos escolares constituyen una de las bases fundamentales sobre las cuales se sustenta la acción educativa. El saber didáctico que progresivamente va produciendo la investigación en educación queda reflejado en diversas fuentes como revistas, monografías, etc.; pero de forma más accesible se visualiza en los libros de textos escolares, cuyos destinatarios son docentes y alumnos. El libro escolar resulta ser un dispositivo privilegiado en los procesos de selección y traducción de los saberes que serán enseñados. Es un material escrito que vuelca en su interior conceptos, modos de hacer, iconografía y datos seleccionados intencionalmente, e incorpora explícita o implícitamente propuestas metodológicas para el aprendizaje de acuerdo al nivel escolar de destino; a las políticas educativas vigentes que emergen en los diseños curriculares; y a los avances de la ciencia y sus procesos de difusión.

Antes de su aparición, en el siglo XIX, la relación libro-educación era considerada casi una ficción. La historia del libro de texto parece seguir un movimiento paralelo y dependiente del sistema educativo que lo contiene, el cuál le otorga su designio. Existen numerosos trabajos de investigación que consideran a este instrumento como su objeto de estudio, en investigaciones históricas, de educación comparada, de exámenes sociológicos, antropológicos, lingüísticos, epistemológicos, pedagógicos, etc., pero a pesar de esta gran cantidad de trabajos no existe una teoría científicamente comprobada sobre los mismos. Ian Westbury (citado en Fernández Reiris, 2004, p.3), juzga que "el uso del libro de texto, en términos holísticos, es un componente escurridizo de la enseñanza desde el punto de vista de la teoría educativa convencional". Las fuertes identidades disciplinares de los sistemas escolares circunscribieron seriamente la forma en que la investigación educativa ha definido sus problemas, de esta manera la mayor cantidad de indagaciones se han encarado por eruditos de distintas disciplinas más que por pedagogos, lo cuál continúa siendo una dimensión pendiente a resolver. En algunos ámbitos se menosprecian los libros de textos en base a polémicas sobre su calidad pedagógica, sus mensajes ideológicos o la supervisión estatal sobre ellos, pero en otros contextos se los considera como los únicos libros obligatorios de la sociedad (Fernández Reiris, 2004, p.2). Este mandato es observado en los principios que prescriben los organismos internacionales de financiamiento para las reformas educativas de varios países, en los cuales figuran como documentos prioritarios que acompañan la transformación de los sistemas educativos.

En particular para textos escolares de Ciencias Naturales y universitarios de Biología existen pocos autores dedicados a su análisis; de los encontrados se puede mencionar el trabajo de Bar (2001) quien propone identificar la recurrencia de la abducción como inferencia ligada al descubrimiento en textos de Biología destinados a estudiantes universitarios. 
Para textos escolares, Mares et al. (2006) presentan una propuesta para analizar las dimensiones vinculadas a la comprensión lectora y transferencia del aprendizaje en libros de Ciencias Naturales de educación primaria; Solarte (2009) propone un análisis de textos escolares de Ciencias Naturales aplicando la teoría de la transposición didáctica; Gallegos y Flores (2003) realizan cuatro estudios sobre libros de Física y Biología; Kesidou (2001) relaciona los textos de Biología con los planes y programas de estudio; Schiefelbein y Farres (1991) como también Staver y Bay (1989) tienen en cuenta si los textos propician el desarrollo de las capacidades intelectuales y lingüísticas; Barrow (2000) junto a Gallegos et al. (2003) analizan si existen correspondencias entre los contenidos conceptuales presentes en los textos y los sistemas conceptuales de la disciplina. En su tesis doctoral, Calderero Hernández (2004) presenta el método de grafos textuales para la interpretación de textos de Ciencias de la Naturaleza utilizados en la enseñanza secundaria y, desde una perspectiva sistémico-funcional, Chamorro y Barletta (2008) proponen el análisis del discurso de textos escolares de Ciencias Naturales.

Estos autores coinciden en mostrar la importancia de promover la participación activa del alumno, dejando espacios para la reflexión; considerando que el texto no debe ser un instrumento cerrado que explicite todo, sino que permita distintos ritmos de aprendizaje y propicie las relaciones entre los contenidos. Además estos modelos de valoración con diferentes orientaciones y distintos puntos de vista pueden atender a una metodología cualitativa, cuantitativa o cuanti-cualitativa.

En investigaciones realizadas para identificar diferentes modos de uso de este medio didáctico se caracterizaron a los docentes en dos grandes grupos: aquellos que dependen extremadamente de los libros de textos y aquellos que presentan una autonomía respecto de los mismos, pero con múltiples facetas determinadas por sus enfoques pedagógicos, por contextos socio institucionales y por la pertenencia social de los estudiantes. A la vez, el uso de los libros varía según las materias de enseñanza y el nivel evolutivo de los alumnos. Desde el punto de vista docente, los libros de texto actúan como un mapa de navegación que reduce la incertidumbre y complejidad de la enseñanza. En este sentido, Eisner (citado en Fernández Reiris, 2003, p.11) concluye que los libros de textos aportarían un código de seguridad que les marca con claridad, tanto a los estudiantes como a sus padres, la dirección y los alcances de lo que se espera de ellos. Desde un enfoque macrosocial, en función de las políticas culturales y educativas se considera al libro de texto como un "elemento del modelo de control remoto de la calidad de la enseñanza“(Doyle, 1990, p. 349, citado en Fernández Reiris, 2003, p. 11), pero desde la visión del docente se concibe como un objeto que atenúa su ansiedad provocada por la realidad de las aulas y lo abriga de las exigencias contrapuestas que la sociedad impone sobre su trabajo. 


\section{Símbolos y representaciones}

El lenguaje matemático utiliza símbolos y representaciones cuya naturaleza y modo de uso ha sido motivo de investigaciones a lo largo de la historia de esta ciencia. Para expresar o representar un concepto matemático se utiliza un conjunto de signos, símbolos y reglas respondiendo a su carácter sistémico, por lo cual según diferentes autores, se habla de un sistema matemático de signos (Kieran y Filloy, 1989), de sistemas de notación (Kaput, 1992), de sistemas semióticos (Duval, 1993), o de sistemas de representación (Castro Martínez, Rico Romero y Romero Albaladejo, 1997). Se consideran sistemas de representación en matemática al lenguaje natural, las figuras y gráficas, las diferentes escrituras simbólicas, las tablas, cuadros y las notaciones algorítmicas. Un ejemplo es el sistema de referencia que puede ser cartesiano o no, en el cual se consideran dos niveles diferentes de representación, el de los conceptos concretos: por ejemplo una función dada por su RGC, y el de las relaciones entre esos conceptos: por ejemplo la función derivada primera de la función anterior, representada en la misma gráfica cartesiana.

El concepto de representación considera dos entidades relacionadas pero funcionalmente separadas: el objeto representante y el objeto representado (Castro Martínez et al., 1997). Una representación puede permitir el acceso al objeto representado cuando se dispone de al menos dos sistemas semióticos diferentes para determinar la representación de ese objeto, o de una situación, o de un proceso y, además tiene que permitir la conversión de un sistema al otro de forma imperceptible. Si las condiciones anteriores no se cumplen se confunde la representación con el objeto representado y además no se logran reconocer dos o más representaciones diferentes del mismo objeto. Por este motivo, es importante distinguir entre actividades que permitan la transformación de las representaciones en el interior de un mismo registro, de aquellas que admitan las transformaciones en distintos registros y requieran coordinación por parte del sujeto que las realiza (Duval, 1999).

En el dominio de la BP y en los soportes de ciencia escolar, tanto se refiera a la dimensión escolar de nivel secundario como en la formación de formadores, los textos se resuelven con un alto porcentaje de la superficie de las páginas utilizando los diversos tipos de información gráfica (Perales y Jiménez, 2002). Las gráficas son objeto de estudio en este caso, y éstas se definen como representaciones que expresan una relación numérica que existe entre dos variables a través de distintos elementos espaciales: barras, líneas, puntos, etc. Según Preece y Janvier (1993), los alumnos tienden a realizar un procesamiento muy ligero y en la mayoría de los casos se limitan a la lectura de los datos y al reconocimiento de aspectos puntuales de las mismas, apareciendo dificultades cuando se les sugiere indagar en niveles más profundos de interpretación de la información representada. Además de leer 
y construir gráficas, los alumnos necesitan utilizarlas para hacer comparaciones, predicciones y buscar patrones o tendencias entre los datos. Estos procedimientos requieren además un conocimiento del contenido abordado o representado en la gráfica, permitiendo así la realización de inferencias a partir de los datos suministrados, lo que implica una interpretación más profunda de la información presentada. La interpretación de una gráfica supone describirla, decir lo que se ve, siguiendo su perfil y, también implica decir el porqué de ese perfil que dependerá de la cantidad de información o conocimiento que posea quien interpreta la gráfica.

En todo proceso comunicacional, y en este caso en particular en los libros de texto, lo que se persigue es lograr representar, es decir hacer presente una cosa (objeto, persona, idea, etc.) con diversos signos y símbolos, de manera tal que una representación es una forma que reemplaza a la realidad. Cuando se hace la sustitución de la cosa con una forma, se modifica, se modela para adaptarla a esa forma que la representa. Por eso la palabra no es nunca un simple rótulo de la cosa, ni el signo visual la copia del objeto. Al respecto, se dice que la función primigenia de los lenguajes es la de construir modelos del mundo.

\section{Modelo ontosemiótico}

Este modelo consiste en abordar la pertinencia referida al lenguaje, la adecuación con relación a sus imágenes y la idoneidad que hace referencia a las distintas dimensiones de los mismos. A continuación se describen cada uno de estos tres aspectos.

\section{Pertinencia}

La pertinencia semiótica está relacionada con el lenguaje utilizado en los libros de texto. Recordemos que la Semiótica se ocupa de la comunicación y la cultura a través del estudio cuidadoso no sólo de la estructura de las formas que se generan con el ejercicio de los lenguajes, sino de la relación que existe entre un emisor que deposita los significados en un texto y un receptor que los retoma y amplía. Desde este enfoque se analiza el lenguaje natural y simbólico que presentan los libros de texto, la clasificación de los signos artificiales y las distintas representaciones gráficas. Se utiliza la taxonomía propuesta por Javier de Lorenzo (1989) para los signos artificiales, y se distingue entre signo estrictamente artificial, signo gráfico único, signo compuesto por varias letras, término, figura y signo artificial.

Para las representaciones gráficas, Postigo y Pozo (2000) distinguen cuatro tipos de información gráfica: los diagramas, que expresan una relación conceptual; las gráficas, una relación cuantitativa; los mapas, una relación espacial selectiva y las ilustraciones para una relación espacial reproductiva. Las relaciones cuantitativas que se expresan utilizando gráficas, se clasifican 
en histogramas, diagramas cartesianos, gráficas cartesianas, diagramas de barras, diagramas de pastel, diagramas de bastones, tablas, diagramas de líneas y diagramas arbolares. Cabe aclarar que un diagrama cartesiano es una representación gráfica en la que se utilizan dos ejes ortogonales, en los cuales se pueden vincular variables cualitativas o cuantitativas; en cambio, una representación en gráficas cartesianas necesariamente vincula variables cuantitativas. A su vez, a las RGC se las clasifica en: diagramas de líneas, de líneas comparativas, de intersección de regiones, de líneas y barras, de puntos, de puntos y líneas, y de líneas y figuras.

\section{Adecuación}

La adecuación corresponde al segundo plano de análisis y se refiere a la propiedad textual basada en el cumplimiento de las normas relacionadas con el tema y la situación que afecta a la constitución de un texto. Perales y Jiménez (2002) presentan bases teóricas para evaluar la adecuación de las ilustraciones en los libros de textos de ciencias y luego proponen una taxonomía y una metodología para su análisis. Estos autores afirman que las ilustraciones son muy importantes porque benefician la comprensión de su contenido, considerándolo como uno de los pilares básicos sobre el cuál se sustenta la acción educativa. Conviene aclarar que una imagen es la representación de seres, objetos o fenómenos y que puede ser de carácter gráfico o mental, en cambio una ilustración es una imagen más especifica de carácter exclusivamente gráfico y que acompaña a los textos escritos con la intención de complementar la información que suministra.

Estos autores definen seis dimensiones: función de la secuencia didáctica en la que aparece la ilustración (para que se emplean las imágenes, en que pasajes del texto se sitúan), iconicidad (que grado de complejidad poseen las imágenes), funcionalidad (que se puede hacer con las imágenes), relación con el texto principal (referencias mutuas entre texto e imagen), etiquetas verbales (textos incluidos dentro de las ilustraciones) y contenido científico que las sustenta (caracterización desde el punto de vista mecánico de las situaciones representadas en las imágenes.). En este estudio, solamente, se trabaja con las dimensiones de iconicidad, funcionalidad y etiquetas verbales, ya que nos informan sobre el grado de complejidad que poseen las imágenes, de la valoración sobre la mayor o menor funcionalidad de estas herramientas expresivas y de los distintos tipos de textos incluidos en las mismas.

\section{Idoneidad}

Corresponde al tercer plano de análisis y para realizarlo se utilizan las investigaciones del análisis ontológico-semiótico de un texto, propuesto por Godino (2002), quienes proponen formular hipótesis sobre aquellos puntos 
críticos de la interacción entre los agentes en los cuales pueden existir vacíos de significación o disparidad de interpretaciones, es decir, sirve para detectar conflictos semióticos (Hjelmslev, 1943). Se consideran seis dimensiones para analizar a la idoneidad: dimensión epistémica, cognitiva, semiótica, mediacional, emocional y ecológica. En este estudio, sólo, se analizan las dimensiones epistémica, semiótica y ecológica.

$\checkmark \quad$ La idoneidad epistémica se refiere al grado de representatividad de los significados institucionales implementados o pretendidos, respecto de un significado de referencia. (Font, Acevedo, Castells y Bolite, 2008). El significado de referencia es un constructo a veces difícil de delimitar porque está implícito en todo el proceso y es el resultado de diversos componentes: del significado del objeto en la institución, de la evolución epistémica de dicho objeto, de las orientaciones curriculares, de los diferentes libros de textos, de los significados conceptuales y didácticos personales del docente, etc. Por lo tanto en una investigación se adoptan criterios que delimitan el sistema de prácticas que definen el significado de referencia adecuado a la situación analizada. Es decir, para valorar la idoneidad epistémica de un proceso de instrucción realizado (significado implementado) o de un proceso planificado en un libro de texto (significado pretendido) es necesario establecer primero el significado de referencia que sirva de comparación. Para valorar la idoneidad epistémica de un texto que utiliza lenguaje matemático., se consideran los siguientes indicadores: lenguaje, situaciones, procedimientos, definiciones, proposiciones y argumentos.

$\checkmark \quad$ La idoneidad semiótica o interaccional se refiere al grado en que las trayectorias o configuraciones didácticas permiten identificar posibles conflictos semióticos, los cuales pueden ser detectados a priori, y son denominados conflictos potenciales; o los detectados durante el proceso de instrucción mediante la negociación de significados que corresponden a los conflictos efectivos y residuales. En este trabajo sólo se consideran los conflictos que pueden presentarse en el análisis de los textos, es decir los conflictos semióticos denominados potenciales, con respecto al lenguaje, a las imágenes y a la idoneidad del texto en cuestión.

$\checkmark \quad$ La idoneidad ecológica describe el grado de adaptación curricular, del proceso de estudio, a las condiciones del entorno social-profesional, a la apertura hacia la innovación, a las directrices en política educativa, etc., en que se implementa. Se consideran también, en este caso, las relaciones que se establecen con otros contenidos definidos dentro de la BP, como en el interior de la propia Biología, y con aquellas disciplinas afines a los mencionados contenidos. Para la valoración de la idoneidad ecológica, se consideran los siguientes indicadores: adaptación al currículo y conexiones intra e interdisciplinares. 
Lo expresado anteriormente podrá visualizarse, aunque no de modo profundo ni general en la producción de un libro de texto, en este caso es necesario atender a la textura que el autor construye al seleccionar las palabras, los íconos y las relaciones recíprocas que éstos pueden establecer y la invitación al diálogo por parte del potencial lector.

En la Tabla 1 se sintetizan los indicadores específicos utilizados en el análisis de los libros de texto realizado en este trabajo.

Tabla 1. Indicadores analizados en los libros de texto

\section{PERTINENCIA}

1.1 Número de signos naturales y número de signos artificiales

1.2 Clases de signos artificiales

1.2.1 Signos gráficos únicos $(\mathrm{A}, \mathrm{B}, \mathrm{C} \ldots)$

1.2.2 Signos compuestos por varias letras (ADN, km, $\mathrm{cm}, \mathrm{ARN}, \mathrm{AA}$, aa, a.C., km, $\mathrm{m} / \mathrm{s}^{2}, \mathrm{NO}_{3}, \mathrm{SO}_{2}, \mathrm{CH}_{2} \mathrm{O}, \mathrm{CO}_{2}$ )

1.2.3 Figuras

1.2.4 Signos artificiales $\left(0,1,2,3, \ldots, \mathrm{I}, \mathrm{II}, \mathrm{III}, \mathrm{IV}, \mathrm{V}, \ldots, \mathrm{X}, \ldots, 1^{\circ}, 2^{\circ}, \ldots, \%, \mathrm{x},+,-\right.$, $=, /, 1 / 4,1 / 2)$

1.3 Clases de representaciones gráficas

1.3.1 Diagramas: Esquemas, mapas conceptuales, cuadros sinópticos, organigramas, diagramas de flujo.

1.3.2 Gráficas: Histogramas, diagramas y representaciones gráficas cartesianas, diagramas de barras, diagramas de sectores, diagramas de bastones, tablas, diagramas de líneas, diagramas de árbol.

1.3.3 Mapas: Mapas geográficos, planos, croquis, dibujos esquemáticos, pirámides tróficas.

1.3.4 Ilustraciones: Fotografías, dibujos.

1.4 Clases de RGC:

Diagramas de líneas, de líneas comparativas, de intersección de regiones, de líneas y barras, de puntos, de puntos y líneas, y de líneas y figuras.

2. ADECUACIÓN (de las Ilustraciones)

2.1 Grado de iconicidad

2.1.1 Fotografías

2.1.2 Dibujos figurativos

2.1.3 Dibujos figurativos + signos

2.1.4 Dibujos figurativos + signos normalizados

2.1.5 Dibujos esquemáticos

2.1.6 Dibujos esquemáticos + signos

2.1.7 Descripción en signos normalizados

2.2 Funcionalidad
2.2.1 Inoperantes
2.2.2 Operativas elementales
2.2.3 Sintácticas

2.3 Etiquetas verbales

2.3.1 Sin etiquetas

2.3.2 Nominativas

2.3.3 Relacionales

3. IDONEIDAD

3.1 Idoneidad epistémica

3.1.1 Lenguaje

3.1.2 Situaciones (ejercicios, problemas, cuestionarios y experiencias de laboratorio)

3.1.3 Acciones (operaciones, algoritmos, técnicas de cálculo)

3.1.4 Conceptos de BP (evolución, especie, población)

3.1.5 Leyes Biológicas 


\subsubsection{Argumentos}

3.2 Idoneidad semiótica (conflictos potenciales inter e intralingüísticos)

3.3 Idoneidad ecológica

3.3.1 Adaptación al currículo

3.3.2 Conexiones intra e interdisciplinares

\section{Metodología}

Se aplicó el análisis ontosemiótico a diez propuestas editoriales, de Ciencias Naturales para Educación Secundaria, más utilizadas en los distintos establecimientos educativos (Tabla 2), y a ocho propuestas editoriales de textos de Biología demandados en los programas de formación en las carreras de profesorado de Biología en la provincia de Mendoza (Tabla 3).

Tabla 2. Libros de texto de Ciencias Naturales, incluidos en el estudio

\begin{tabular}{|c|c|}
\hline $\begin{array}{l}\text { Texto } \\
\mathrm{N}^{\mathrm{o}}\end{array}$ & Referencia bibliográfica \\
\hline 1 & $\begin{array}{c}\text { Abellan, K., Bazo, R., Caro, G. \& Selles-Martínez, J. (2007). Ciencias Naturales. } \\
\text { Buenos Aires: Tinta Fresca. }\end{array}$ \\
\hline 2 & $\begin{array}{c}\text { Aletti, S., Bosack, A. \& Fernández, M. (2000). Ciencias Naturales - 8. Buenos Aires: } \\
\text { Santillana. }\end{array}$ \\
\hline 3 & $\begin{array}{c}\text { Aristegui, R., Barderi, M. \& Bosack, A. (2001). Ciencias Naturales - 9. Buenos } \\
\text { Aires: Santillana. }\end{array}$ \\
\hline 4 & $\begin{array}{c}\text { Carreras, N., Conti, O., Fernández, C. \& Lantz, M. (2001). Ciencias Naturales - } 8 . \\
\text { Buenos Aires: Puerto de Palos. }\end{array}$ \\
\hline 5 & $\begin{array}{l}\text { Carreras, N., Conti, M. \& Milano, C. (2001). Ciencias naturales 9. Buenos Aires: } \\
\text { Puerto de Palos. }\end{array}$ \\
\hline 6 & $\begin{array}{c}\text { Cerdeira, S., Cwi, M.; Ferrari, H. \& Greco, M. (2004). Ciencias Naturales y } \\
\text { Tecnología -9. Buenos Aires: Aique. }\end{array}$ \\
\hline 7 & $\begin{array}{c}\text { Costaguta, M., Frid, D. \& Sturla, A. (2004). El libro de la naturaleza - 9. Buenos } \\
\text { Aires: Estrada. }\end{array}$ \\
\hline 8 & $\begin{array}{c}\text { Hunell, J., Leschintta, M. \& Rela, A. (2003). Atomo. Ciencias Naturales 8. Buenos } \\
\text { Aires: SM. }\end{array}$ \\
\hline 9 & $\begin{array}{l}\text { Hunell, J., Leschintta, M., Rela, A. \& Tignanelli, H. (2003). Atomo. Ciencias } \\
\text { Naturales 9. Buenos Aires: SM. }\end{array}$ \\
\hline 10 & Jáuregui, S. (2004). El libro de la naturaleza - 9. Buenos Aires: Estrada \\
\hline
\end{tabular}

Tabla 3. Libros de texto de Biología de formación de Profesorado, incluidos en el estudio

\begin{tabular}{cc}
\hline Texto $\mathrm{N}^{\mathrm{o}}$ & Referencia bibliográfica \\
\hline 1 & Audersik, T \& Audersik, G. (1997). Biología. La vida en la Tierra. México: \\
& Prentice Hall- Hispanoamericana. \\
2 & Campbell, N. \& Reece, J. (2007). Biología. Buenos Aires: Médica panamericana. \\
3 & Curtis, H., Barnes, N., Schnek, A. \& Massarini, A. (2008). Biología. Buenos Aires: \\
& Médica panamericana. \\
4 & Fried, G. (1994). Biología. Madrid: Mc Graw Hill.
\end{tabular}



la Biología. Buenos Aires: Médica panamericana.

6 Solomon, E., Berg, L. \& Martin, D. (2001). Biología. México: Mc Graw- Hill Interamericana.

En ambos niveles educativos los establecimientos provinciales de gestión privada y pública, son dependientes de la Dirección General de Escuelas (Gobierno de Mendoza) y de la Universidad Nacional de Cuyo. El estudio de tipo exploratorio, descriptivo y comparativo de los libros de texto se centró en los capítulos referidos a BP, y se resolvió en el ciclo lectivo 2012, aplicándolo en las propuestas editoriales enunciadas por las instituciones en el plan de estudio de cada caso. El modelo de análisis, explicado en el apartado 3.c., se muestra en la Figura 1.

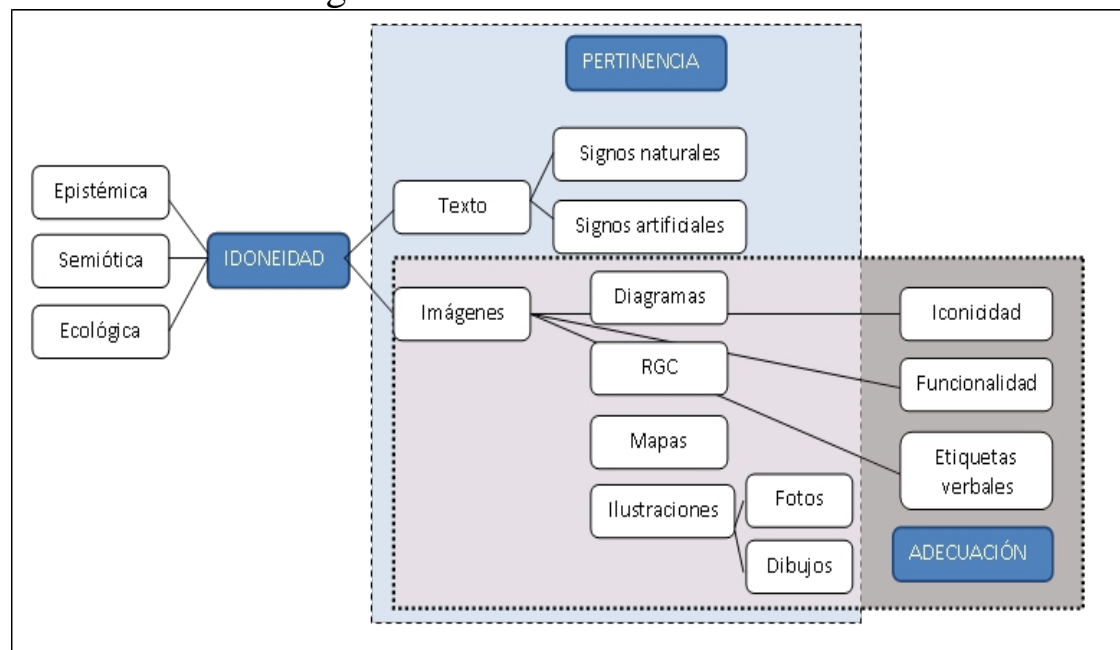

Figura 1. Vinculación de las variables, dimensiones e indicadores aplicados en el análisis ontosemiótico

Para enriquecer a la investigación se recurre a un modelo integrado multimodal (cuantitativo y cualitativo), es decir, desde un enfoque cuantitativo se utiliza el conteo y el uso de la estadística para establecer con exactitud patrones de comportamiento, y desde un enfoque cualitativo basado en la observación, descripción y evaluación del proceso, a fin de establecer suposiciones o ideas en consecuencia (Hernández Sampieri, Fernández Collado y Baptista, 2006). Además para evitar resultados subjetivos por parte del investigador, y para dar fiabilidad al estudio, se acompañó el análisis con imágenes escaneadas de los propios libros de texto para contrastar los resultados y las inferencias obtenidas. 


\section{El estudio en el nivel secundario}

Se resolvió sobre propuestas editoriales enunciadas en los planes de clase y sugeridas para alumnos que cursan $1^{\circ}$ y $2^{\circ}$ año de Educación Secundaria. El grupo diana para estas propuestas corresponde a la franja etaria entre 13 y 15 años. Los contenidos referidos a los conceptos de BP están incluidos en las actuales propuestas curriculares de Educación Secundaria en el Área de Ciencias Naturales, que figuran en el Documento: "Saberes indispensables para el Ciclo básico de la Educación Secundaria, Materiales de acompañamiento al Docente", basado en la propuesta de Núcleos de aprendizajes prioritarios (NAP, 2006) del Ministerio de Educación de la Nación. El documento jurisdiccional fue entregado a docentes de la provincia de Mendoza en el año 2012. En la Tabla 4 se presentan los saberes indispensables, vinculados a conceptos de la BP, correspondientes al eje "Seres vivos: Diversidad, unidad, interrelaciones y cambios", y cuya apertura en conceptos aparece en las propuestas didácticas del tercer nivel de especificación (el aula).

Tabla 4. Saberes indispensables definidos para primero y segundo año de la escolaridad

Secundaria, que se relacionan con BP

\begin{tabular}{|c|c|}
\hline \multicolumn{2}{|c|}{ Saberes Indispensables-Ciencias Naturales } \\
\hline Primer año & Segundo año \\
\hline $\begin{array}{c}\text { - Interpretar las teorías que explican el } \\
\text { origen de la vida y su vínculo con las } \\
\text { características que definen a los seres } \\
\text { vivos. } \\
\text { - Caracterizar la función de relación, } \\
\text { autorregulación y control en los seres } \\
\text { vivos, asociada con los cambios en los } \\
\text { ambientes interno y externo (como en } \\
\text { animales ectodermos y endodermos, } \\
\text { tropismos y nastias en vegetales, por } \\
\text { ejemplo) } \\
\text { - Reconocer la importancia de la } \\
\text { preservación de la biodiversidad desde los } \\
\text { puntos de vista ecológico, evolutivo, } \\
\text { científico y económico. }\end{array}$ & $\begin{array}{l}\text { - Interpretar los mecanismos hereditarios } \\
\text { propuestos por Mendel. } \\
\text { - Reconocer el papel de los cromosomas } \\
\text { en la herencia. } \\
\text { - Interpretar las teorías científicas que } \\
\text { explican la evolución de las especies y la } \\
\text { adaptación de los organismos al ambiente. } \\
\text { - Interpretar algunas temáticas científicas } \\
\text { actuales que generan debates en la sociedad } \\
\text { (clonación, alimentos transgénicos, huellas } \\
\text { de ADN, etc.). }\end{array}$ \\
\hline
\end{tabular}

\section{El estudio en el nivel de profesorado}

El estudio de los textos propuestos para la formación del profesorado en Biología se centró en producciones editoriales de Biología general y Ecología, en ambos casos en los capítulos referidos a BP y que forman parte de la bibliografía obligatoria según programas de las instituciones de formación Superior.

La Ley de Educación Superior N ${ }^{\circ} 24521$ sancionada en julio de 1995, junto a las normativas de la Dirección de Educación Superior dependiente de la Dirección General de Escuelas (nivel jurisdiccional), regulan a la entidad 
educativa formadora quien es la responsable de definir los lineamientos necesarios para la carrera de Profesorado de Educación Secundaria en Biología, proponiendo su propio diseño curricular.

\section{Resultados y Discusión}

\section{Resultados para la pertinencia}

Se realizó un conteo de la cantidad de signos naturales y artificiales que figuran en cada uno de los textos en los capítulos correspondientes a BP. La Tabla 5 muestra ejemplos de signos artificiales y su clasificación extraídos de los textos escolares.

Tabla 5. Ejemplos de signos artificiales extraídos de los textos escolares

\begin{tabular}{|c|c|}
\hline Signos artificiales & Ejemplos de signos hallados en los textos \\
\hline $\begin{array}{l}\text { Signo estrictamente } \\
\text { artificial }\end{array}$ & No se encuentran \\
\hline Signo gráfico único & $\mathrm{A}, \mathrm{B}, \mathrm{C}, \ldots$ \\
\hline $\begin{array}{l}\text { Signo compuesto por } \\
\text { varias letras }\end{array}$ & $\begin{array}{l}\mathrm{ADN}, \mathrm{km}, \mathrm{cm}, \mathrm{ARN}, \mathrm{AA}, \text { aa, a.C., km, m/s } \mathrm{s}^{2}, \mathrm{NO}_{3}, \mathrm{SO}_{2}, \\
\qquad \mathrm{CH}_{2} \mathrm{O}, \mathrm{CO}_{2}\end{array}$ \\
\hline Término & No se encuentran \\
\hline Figura & \\
\hline Signo artificial & 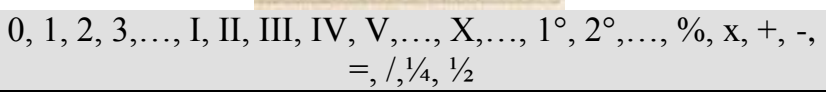 \\
\hline
\end{tabular}

Del mismo modo se realizó el conteo para las distintas representaciones gráficas que se ejemplifican en la Tabla 6 .

Tabla 6. Gráficas contempladas en los libros de textos escolares

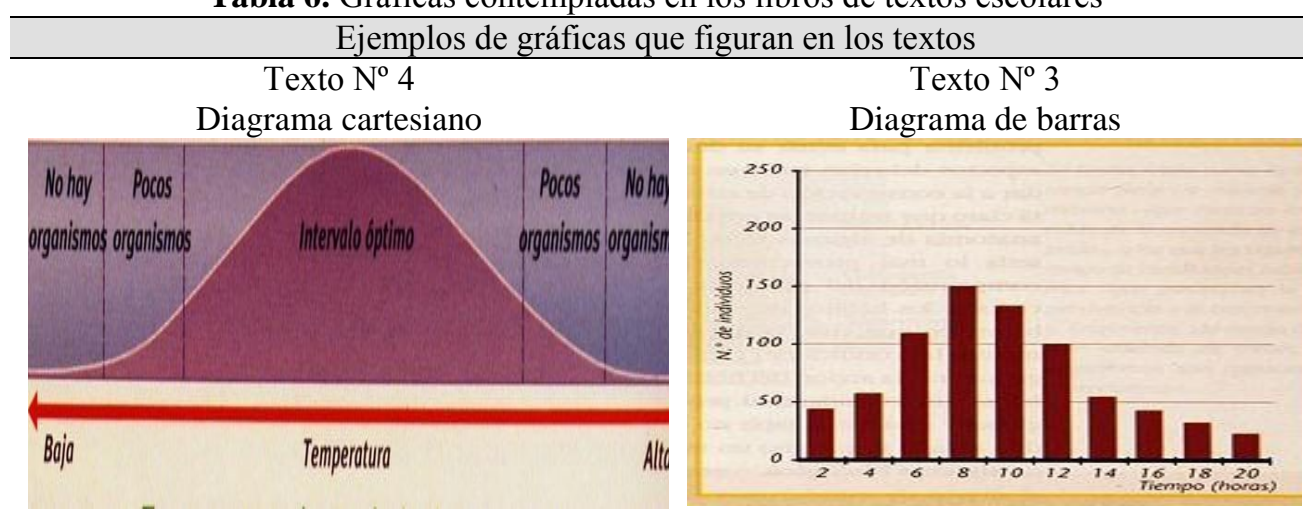




\section{Resultados para la adecuación}

La Tabla 7 muestra algunos ejemplos de dibujos utilizados en el análisis de la iconicidad, funcionalidad y etiquetas verbales.

Tabla 7. Dibujos extraídos de los libros de textos escolares

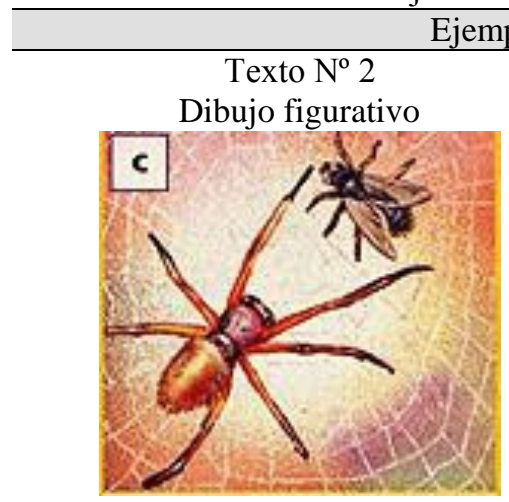

Texto $\mathrm{N}^{\circ} 8$

Dibujo figurativo+signos normalizados

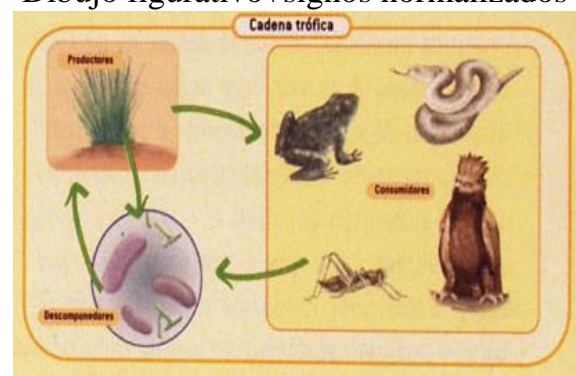

Ejemplos para la funcionalidad

Texto $\mathrm{N}^{\circ} 2$

Ilustraciones inoperantes

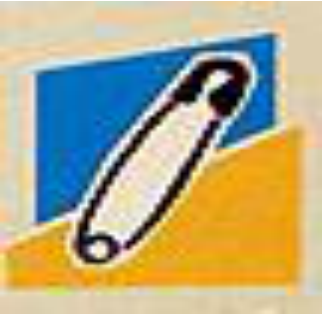

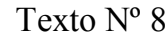

Ilustraciones sintácticas

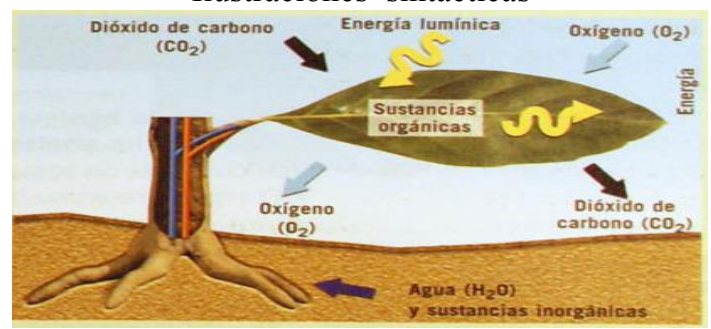

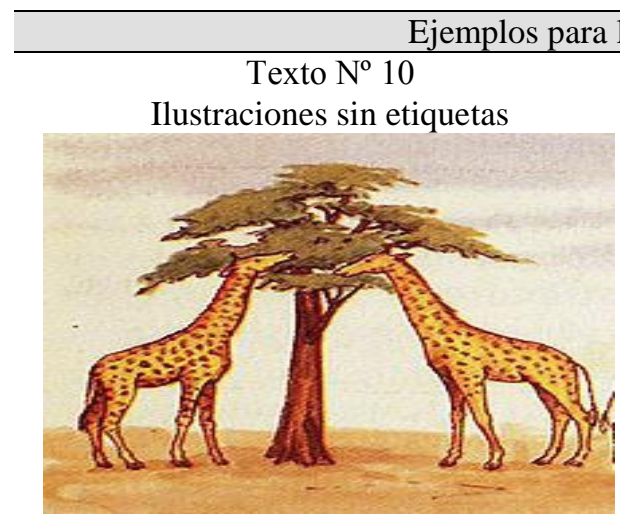

Texto $\mathrm{N}^{\circ} 4$

Ilustraciones nominativas

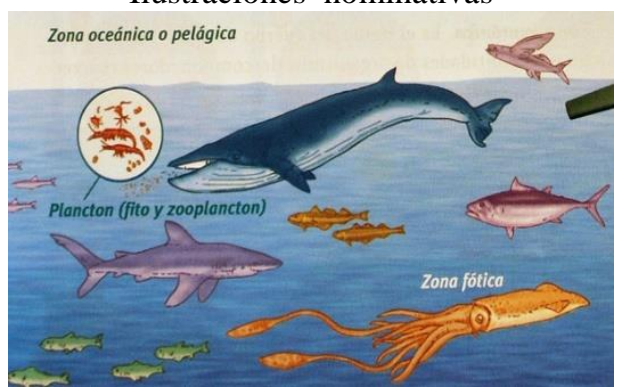

Los principales resultados obtenidos de la dimensión Pertinencia y Adecuación, se presentan en la Tabla 8, identificando diferencias y similitudes entre los textos de los distintos niveles educativos. 
Tabla 8. Resultados obtenidos en el análisis ontosemiótico desde las dimensiones pertinencia y la adecuación: diferencias y similitudes

\begin{tabular}{cc} 
Textos de Educación & Textos de Formación de \\
Secundaria & Profesorado \\
\hline
\end{tabular}

Se utiliza un lenguaje científico-técnico en todos los textos

Pertinencia de signos y gráficas

Signos

artificiales

Representaciones gráficas (según

Tabla 1, 1.3.1.)

Gráfica (según Tabla 1, 1.3.2.)

RGC (del punto

1.3.2, específicamente esta representación)

\section{Adecuación de las ilustraciones}

Iconicidad

Funcionalidad

\section{Ilustraciones: $88 \%$ y las gráficas sólo el 4\%.}

Gráficas del tipo tablas de valores (62\%); diagramas cartesianos $24 \%$ y los de árbol $10 \%$; histogramas y diagramas de bastones: $0 \%$

RGC: $0 \%$.

Del bajo porcentaje de diagramas cartesianos hallados, sólo se utilizan los diagramas de líneas (71\%) y los de líneas comparativas (29\%). Las demás clasificaciones no figuran en los textos analizados.

$50 \%$ (aproximadamente) de la superficie de sus páginas.

Fotografías es el lenguaje icónico de mayor porcentaje. Función decorativa Dibujos figurativos: $23 \%$ Dibujos esquemáticos+signos: $4 \%$

Ilustraciones inoperantes: $42 \%$ Ilustraciones operativas: $0 \%$
Mayor presencia y variedad. Los más abundantes son los signos más cercanos al lenguaje natural. Los números arábigos $(0,1,2, \ldots)$, los números romanos (IV, IX, ...), los números ordinales y fraccionarios.

Ilustraciones: $60 \%$, las gráficas se presentan en un $25 \%$.

Diagramas cartesianos: 39\%; diagramas de barras $26 \%$; histogramas y diagramas de bastones: $0 \%$.

RGC: $18 \%$.

Figuran todas las categorías analizadas. Los diagramas de líneas comparativas ocupan el mayor porcentaje $(43 \%)$, seguidos de los de líneas (26\%), y líneas y

figuras $(16 \%)$. La menor frecuencia $(1 \%)$ corresponde a los diagramas de intersección de regiones.

$25 \%$ de la superficie de sus páginas

Fotografías es el lenguaje icónico de mayor porcentaje. Función decorativa

Dibujos figurativos: $8 \%$ Dibujos esquemáticos+signos: $10 \%$

Ilustraciones inoperantes: 0\% Ilustraciones operativas: $90 \%$ 


$\begin{array}{ccc}\text { Etiquetas } & \text { Ilustraciones sin etiquetas: } 38 \% & \text { Ilustraciones sin etiquetas: 5\% } \\ \text { verbales } & \text { Ilustraciones con etiquetas } & \text { Ilustraciones con etiquetas } \\ & \text { relacionales: } 45 \% & \text { relacionales: } 81 \%\end{array}$

En la Tabla 9, se avanza en el análisis comparativo de los libros de textos para ambos niveles educativos, atendiendo a la dimensión idoneidad.

Tabla 9. Resultados obtenidos en el análisis ontosemiótico desde la dimensione idoneidad: diferencias y similitudes

Textos de Educación Secundaria

Textos de formación de Profesorado

Idoneidad

epistémica

Cada texto posee una diagramación diferente para presentar las distintas actividades o situaciones y los cuestionarios ocupan el mayor porcentaje, seguido de los problemas y experiencias de laboratorio.

No se utiliza la notación científica, ni la nomenclatura

binomial. No presentan operaciones, ni algoritmos, tampoco diferentes técnicas de cálculo o procedimientos, ni tampoco cambios de registro.

No se hace referencia explícita de principios o de leyes biológicas.

Los argumentos lógico-racionales se utilizan en todos los textos, como por ejemplo razonamientos por analogía, por generalización o sintomáticos.

Los conceptos de "evolución", "especie” y "población" se definen de diferentes formas y en algunos textos sólo se los menciona.

Idoneidad semiótica
Los posibles conflictos semióticos se vinculan al uso de la notación simbólica de compuestos químicos, a los símbolos que presentan las ilustraciones sintácticas, o a los diagramas cartesianos que carecen de leyendas.
Se detectan posibles conflictos semióticos en el uso de la notación simbólica referida a los conceptos de límite funcional y función derivada; en las ilustraciones sintácticas, como también vinculados a la interpretación biológica de RGC en las cuales se utilizan más de dos variables o se presentan datos solapados. 


$\begin{array}{ccc}\text { Idoneidad } & \text { Se adecuan a los contenidos } & \text { Se ajustan a los contenidos } \\ \text { ecológica } & \text { curriculares definidos para este } & \text { curriculares definidos para los } \\ & \text { nivel educativo, sin embargo, se } & \text { profesorados de Biología según la } \\ \text { observan algunas falencias en la } & \text { Ley de Educación Superior en } \\ \text { presentación y tratamiento de } & \text { Argentina, aunque se observan } \\ \text { algunos contenidos, o relaciones } & \text { diferencias en su presentación y } \\ & \text { interdisciplinares. } & \text { tratamiento. }\end{array}$

\section{Conclusiones}

Desarrollar en el estudiante la capacidad de interactuar con conciencia en el medio ambiente, reconociendo " la importancia de la preservación de la biodiversidad desde los puntos de vista ecológico, evolutivo, científico y económico" demanda conceptualizaciones que reclaman estructurar esquemas mentales fuertes. Analizar principios e hipotetizar probabilidades de las poblaciones biológicas requiere sin dudas la interacción con diversas representaciones que demanden interpretar la interrelación de variables y la idea de modelo para enunciar, describir, argumentar explicaciones provisorias o conclusiones de casos.

Los libros de textos correspondientes al nivel de escolaridad secundaria o media, en los capítulos indicados como objeto de estudio, se adecuan a los contenidos curriculares definidos para este nivel educativo. En ellos se utiliza mayoritariamente el lenguaje verbal basado en signos naturales. Entre las imágenes, las ilustraciones son las más abundantes, y dentro de ellas, las fotografías, lo que muestra la tendencia a buscar un alto grado de iconicidad y bajo nivel de abstracción. Las gráficas en cambio son muy escasas, y en casi la mitad de los manuales no aparece absolutamente ninguna. Las pocas gráficas encontradas son fundamentalmente tablas, diagramas cartesianos -de líneas y de líneas comparativas- y diagramas de árbol.

Conviene destacar que, frente a una adecuación ecológica aceptable, hay una evidente deficiencia en la idoneidad semiótica, lo que podría ser fuente de conflictos semióticos potenciales asociados a diagramas cartesianos que carecen de leyendas o se presentan desvinculados del texto explicativo, dificultando su lectura, interpretación y uso. Las propuestas para la interacción con las RGC potenciando la interpretación y predicción de dinámicas poblacionales, intercambios en la comunidad o la variabilidad en las poblaciones, son escasas o nulas.

Los libros de textos de Profesorado de Biología analizados, en coincidencia con los textos escolares, utilizan también un lenguaje mayoritariamente de signos naturales. Las ilustraciones ocupan casi el veinticinco por ciento de la superficie de sus páginas; aunque expresan una distribución más equitativa entre fotografías y dibujos, lo que muestra un menor grado de iconicidad con respecto a los de nivel secundario. No se usan las ilustraciones inoperantes. Se observa un notorio aumento del uso de las 
gráficas, en su mayoría diagramas y gráficos cartesianos y diagramas de barras, y se observan conversiones de estas representaciones, lo que favorece la interpretación del lector. Se favorece una interacción positiva con el potencial lector, a partir de RGC que demandan relacionar la información desde diversos indicadores.

Es factible concluir que los textos universitarios presentan un lenguaje con mayor capacidad semiótica que los escolares. Particularmente, la relación entre texto e imágenes es más potente; y las imágenes son mas variadas, funcionales y operativas. La variedad también se manifiesta en las gráficas propiamente dichas, donde se fomenta la conversión entre las mismas. Podría afirmarse por tanto que los libros de textos universitarios son, frente a los de educación secundaria, más favorecedores de las interacciones semióticas inter-lingüísticas, y por tanto, de las relaciones entre el lenguaje matemático y biológico. Conviene advertir no obstante que, aunque no se haya podido demostrar en este trabajo por las limitaciones de espacio, el extremo que se acaba de concluir no implica una homogeneidad entre ejemplares, sino que es más bien una tendencia central originada por una alta diversidad de tratamientos.

\section{References:}

1. Arias, C., Leal, L. \& Organista, M. (2011). La modelación de la variación, un análisis del uso de las graficas cartesianas en los libros de texto de Biología, Física y Química de secundaria. Revista de Ciencias. 15, 93-128.

2. Argentina, Ministerio de Cultura y Educación de la Nación, Consejo Federal de Cultura y Educación (1995).Ley de Educación Superior N ${ }^{\circ}$ 24.521 .

3. Argentina, Ministerio de Cultura y Educación de la Nación. Gobierno de la Ciudad de Buenos Aires. (2009). Contenidos para el Nivel Medio. Biología.

4. Bar, A. (2001). Biología y abducción. Un caso de análisis: El texto universitario. http://www1.unne.edu.ar/cyt/2001/2-Humanisticas/H029.pdf

5. Barrow, L. (2000). Do elementary science methods textbooks facilitate the understanding of magnet concepts? Journal of Science and Technology, 9(3), 199-205.

6. Calderero Hernández, J. (2004). Estudio de libros de texto de ciencias de la naturaleza mediante análisis cuantitativo basado en la teoría de grafos. Tesis Doctoral. 
7. Castro Martínez, E., Rico Romero, L. y Romero Albaladejo, I. (1997).Sistemas de representación y aprendizaje de estructuras numéricas. Enseñanza de las Ciencias, 1997, 15(3), 361-371.

8. Chamorro, D. y Barletta, N. (2008). El lenguaje del texto escolar de Ciencias Naturales: problemas para el aprendizaje. Bio-grafia: Escritos sobre la Biología y su Enseñanza. 2(1) ISSN 2027-1034.

9. De Lorenzo, J. (1989). 'La Matemática y el Ámbito conceptual'. Revista de Filosofia M, 3. ép. 1: 43-53.

10. Duval, R. (1993). Registros de representación semiótica y funcionamiento cognitivo del pensamiento. En Hitt, F. (Ed), Investigaciones en Matemática Educativa II (173-201). Grupo Editorial Iberoamérica, México.

11. Duval, R. (1999). Semiosis y pensamiento humano. Registros semióticos y aprendizajes intelectuales. Colombia: Universidad del valle. Instituto de educación y pedagogía. Grupo de educación matemática.

12. Fernández Reiris, A. (2004) .El libro y su interrelación con otros medios de enseñanza. Buenos Aires: Miño y Dávila.

13. Font, V.; Acevedo, J.; Castells, M y Bolite J. (2008). Metáforas y ontosemiótica. El caso de la representación gráfica de funciones en el discurso escolar. Acta Latinoamericana de Matemática Educativa, 21. México.

14. Gallegos, L. y Flores, F. (2003). "Concepciones, cambio conceptual, modelos de representación e historia y filosofía, en enseñanza de la ciencia”, en López y Mota (ed.) Saberes científicos, humanísticos y tecnológicos: procesos de enseñanza y aprendizaje. México: COMIE.

15. García García, J. (2005). La comprensión de las representaciones graficas cartesianas presentes en los libros de texto de Ciencias Experimentales, sus características y el uso que se hace de ellas en el aula. Tesis Doctoral. Universidad de Granada.

16. Godino, J.D. (2002). Un enfoque ontológico y semiótico de la cognición matemática. Recherches en Didactique des Mathématiques, 22 (2.3), 237-284.

17. González Astudillo, M. y Sierra, M. (2004). Metodología de análisis de libros de texto de Matemáticas. Los puntos críticos en la enseñanza secundaria en España durante el siglo XX. Enseñanza de las Ciencias, 2004, 22(3), 389-408. 
18. Hernández Sampieri, R., Fernández Collado, C. y Baptista, P. (2006). Metodología de la investigación. MacGraw - Hill. Interamericana. México.

19. Hjelmslev, L. (1943). Prolegomena to a Theory of Language. University of Wisconsin.

20. Jiménez Tejada, M., González-García, F. y Hódar, J.A. (2008). El aprendizaje del concepto biológico de población: cómo pueden las ciencias sociales y las matemáticas colaborar con la didáctica de la biología. Didáctica de las Ciencias Experimentales y Sociales, 22 (1): 103-114.

21. Jiménez Tejada, M. (2009). Los conceptos de población y de especie en la enseñanza de la biología: concepciones, dificultades y perspectivas. Tesis doctoral. Universidad de Granada.

22. Jiménez Tejada, M., Sanchez Monsalve, C. y Gonzalez Garcia, F. (2013). How Spanish primary school students interpret the concepts of population and species, Journal of Biological Education, 47:4, 232239, DOI: 10.1080/00219266.2013.79908.

23. Kaput, J. (1992). Technology and mathematics education, en Grouws, D.A. (ed.). Handbook of Research on Mathematics Teaching and Learning (515-556). New York: MacMillan P.C.

24. Kesidou, S. (2001). Aligning curriculum material with national science standards: The role of Project 2061's curriculum-material analysis procedure in professional development. Journal of Science Teacher Education, 12, 47-65.

25. Kieran, C. y Filloy Yagüe, E. (1989). El aprendizaje del Algebra escolar desde una perspectiva psicológica. Enseñanza de las Ciencias, 1989, 7(3), 229-240.

26. Mares, G., Rivas, O. y otros. (2006). Análisis de lecciones de enseñanza de biología en primaria: propuesta para analizar los libros de texto de ciencias naturales. Revista mexicana de investigación educativa. ISSN 1405- 6666, 11 (30), 883-911.

27. Mendoza, Dirección General de Escuelas (2012). Saberes indispensables para el Ciclo Básico de la Educación Secundaria. Materiales de acompañamiento al docente. 
28. Perales Palacios, F. J. y Jiménez, J. (2002). Las ilustraciones en la enseñanza-aprendizaje de las Ciencias. Análisis de libros de textos. Enseñanza de las Ciencias, 20 (3), 369-386.

29. Pimm, D. (1990). El lenguaje matemático en el aula. Madrid: Morata 30. Postigo Y. y Pozo, J. (1999). El aprendizaje estratégico: enseñamos a aprender desde el currículo. Madrid: Santillana.

31. Postigo Y. y Pozo, J. (2000). Cuando una gráfica vale más que 1000 datos: la interpretación de gráficas por alumnos adolescentes. Universidad autónoma de Madrid.

32. Pozo, J. y Flores, F. (Coords.) (2007). Cambio conceptual y representacional en el aprendizaje y la enseñanza de la ciencia. Madrid: Antonio Machado. (pp. 107-124)

33. Preece, J. y Janvier, C. (1993). Interpreting trends in multiple-curve graphs of ecological situations: The role of context. International Journal Science Education, 15(2), 199-212.

34. Schiefelbein, E. y Farrés, P. (1991). Evaluación formativa de libros de texto de educación primaria. Revista Latinoamericana de Estudios Educativos (México), XXI (2), 63-87.

35. Staver, J. y Bay, M. (1989). Analysis of the conceptual structure and reasoning demands of elementary science texts at the primary (K-3) level. Journal of Research in Science Teaching, 26, 329-349.

36. Solarte, M. (2009). Análisis de contenidos en los textos escolares de ciencias naturales, aplicando la teoría de la transposición didáctica. Asociación Colombiana para la investigación en Ciencias y Tecnología EDUCyT, Memorias, I congreso Nacional de investigación en educación en ciencias y tecnología, 2009, Junio 22 a 26, ISBN: 978-958-99491-0-8 